3. In der $(\mathrm{C}-\mathrm{H})$-Gruppe auch der reinen Kohlenwasserstoffe ist das positive Ende der Bindungspolarisation zum Wasserstoff gerichtet.

4. Die tatsächlich beobachtete Senkung der Valenz-Frequenz einer $(\mathrm{C}-\mathrm{H})$-Gruppe beim Übergang von der Gas-Phase in eine Flüssigkeit mit AkzeptorEigenschaften kann unmöglich ein Maß für die Energie der Wasserstoffbrücke sein.
5. Die $(\mathrm{C}-\mathrm{H})$-Gruppen von $\mathrm{CD}_{3} \cdot \mathrm{CHCl} \cdot \mathrm{CD}_{3}$ und von $\mathrm{Cl}_{2} \mathrm{CH} \cdot \mathrm{CH}_{3}$ sind praktisch keine Protonendonatoren.

Herrn Professor Dr. K.-H. Hellwege danken wir für förderndes Interesse, den Herren Dr. H. Bastin und Dr. H. Тн. Heimes für klärende Diskussionen.

\title{
Theory and Calculation of Centrifugal Distortion Constants for Polyatomic Molecules
}

\author{
S. J. Cyvin, B. N. Cyvin and G. Hagen \\ Institute of Physical Chemistry, Technical University of Norway, Trondheim, Norway \\ (Z. Naturforsch. 23 a, 1649-1655 [1968] ; received 27 June 1968)
}

\begin{abstract}
The method of Kivelson and Wilson for calculating centrifugal distortion constants $\left(t_{\alpha \beta \gamma \delta}\right)$ is treated in terms of matrix notation. A modified method is developed, by which the quantities of $t_{\alpha \beta \gamma \delta}$ are obtained with the aid of certain elements $T_{\alpha \beta, S}^{(i)}$, rather than the partial derivatives of inertia tensor components, $J_{\alpha \beta, S}^{(i)}$, used in the method of Kivelson and Wilson. The $T_{\alpha \beta, S}^{(i)}$ elements may easily be evaluated for a given molecular model from the equilibrium position vectors and the Wilson $s$ vectors. The bent symmetrical $X Y_{2}$ molecule model is used for exemplification of the developed theory. Also included are some numerical results for a number of molecules of the bent symmetrical $\mathrm{XY}_{2}$ type.
\end{abstract}

The theory of centrifugal distortion constants has been developed by KIVELson and WiLson ${ }^{1}$. The aims of the present work are the following. (i) Reformulation of the theory by extensive employment of matrix notation. To this step we have been inspired by Meal and PoLo's ${ }^{2}$ successful matrix formulation of the theory of Coriolis coupling of vibration-rotation. (ii) Simplification of the method for practical calculations, specifically as outlined in the next point. (iii) Developing relations for centrifugal distortion constants in terms of certain quantities which should be suitable for tabulation for the various molecular models. (iv) Tabulation of such quantities as mentioned in the preceding point. (v) Exemplification of the theory by numerical results for specific molecules.

\section{Survey of the Theory}

We shall concentrate the attention upon the computation of the quantities of $t_{\alpha \beta \gamma \delta}$ in the notation of KIVELSON and WILSON ${ }^{1}$, viz.

$$
t_{\alpha \beta \gamma \delta}=-2 I_{\alpha \alpha}^{\mathrm{e}} I_{\beta \beta}^{\mathrm{e}} I_{\gamma \gamma}^{\mathrm{e}} I_{\delta \delta}^{\mathrm{e}} \tau_{\alpha \beta \gamma \delta} .
$$

1 D. Kivelson and E. B. Wirson, Jr., J. Chem. Phys. 21, 1229 [1953].
Here $\alpha, \beta, \gamma, \delta=x, y$ or $z$; and $I_{x x}^{\mathrm{e}}, I_{y y}^{\mathrm{e}}$ and $I_{z z}^{\mathrm{e}}$ are the principal moments of inertia at equilibrium. The considered quantities are given by

$$
\begin{gathered}
t_{\alpha \beta \gamma \delta}=\sum_{k} J_{\alpha \beta, Q}^{(k)} J_{\gamma \delta, Q}^{(k)} \sigma_{k} \\
t_{\alpha \beta \gamma \delta}=\sum_{i} \sum_{j} J_{\alpha \beta, S}^{(i)} J_{\gamma \delta, S}^{(j)} N_{i j},
\end{gathered}
$$

where the latter formula (3) is equivalent to Eq. (17) of KIVELson and Wilson ${ }^{1}$. In our Eq. (2) $\sigma_{k}$ is used to denote the frequency parameters

$$
\sigma_{k}=1 / \lambda_{k}=1 /\left(4 \pi^{2} c^{2} \omega_{k}^{2}\right),
$$

where $c$ is the velocity of light, and $\omega_{k}$ represents the vibrational frequencies (in wave numbers). $N_{i j}$ in Eq. (3) denotes the element of the inverse forceconstant matrix (compliance constant ${ }^{3}$ ) corresponding to the $S_{i} S_{j}$ product of internal coordinates. The $J$ quantities are partial derivatives of the instantaneous inertia tensor components, viz.

$$
J_{\alpha \beta, Q}^{(k)}=\left(\frac{\partial I_{\alpha \beta}}{\partial Q_{k}}\right)_{0}, J_{\alpha \beta, S}^{(i)}=\left(\frac{\partial I_{\alpha \beta}}{\partial S_{i}}\right)_{0} .
$$

2 J. H. Meal and S. R. Polo, J. Chem. Phys. 24, 1119, 1126 (1956).

3 J. C. Decius, J. Chem. Phys. 38, 241 [1963]. 
The partial derivatives are taken at equilibrium where all the internal coordinates $\left(Q_{k}, S_{i}\right)$ vanish, since they designate displacements from equilibrium. Below we also give the inertia tensor components, which are the instantaneous moments of inertia, $I_{\alpha \alpha}$, and products of inertia, $I_{\alpha \beta}$.

$$
\begin{aligned}
I_{x x}=\sum_{a} m_{a}\left(Y_{a}^{2}+Z_{a}^{2}\right), & I_{y z}=-\sum_{a} m_{a} Y_{a} Z_{a}, \\
I_{y y}=\sum_{a} m_{a}\left(Z_{a}^{2}+X_{a}^{2}\right), & I_{z x}=-\sum_{a} m_{a} Z_{a} X_{a},(6) \\
I_{z z}=\sum_{a} m_{a}\left(X_{a}^{2}+Y_{a}^{2}\right), & I_{x y}=-\sum_{a} m_{a} X_{a} Y_{a} .
\end{aligned}
$$

Here $m_{a}$ denotes the mass of atom $a$, and $\left(X_{a}, Y_{a}\right.$, $Z_{a}$ ) identifies the instantaneous position vector for the same atom.

Next we shall develop the partial derivates with respect to an internal coordinate, say $S_{i}$, invoking the cartesian displacement coordinates; $x_{a}, y_{a}, z_{a}$. If $\left(X_{a}^{\mathrm{e}}, Y_{a}^{\mathrm{e}}, Z_{a}^{\mathrm{e}}\right)$ represents the equilibrium position vector of atom $a$, we have

$X_{a}=X_{a}^{\mathrm{e}}+x_{a}, \quad Y_{a}=Y_{a}^{\mathrm{e}}+y_{a}, \quad Z_{a}=Z_{a}^{\mathrm{e}}+z_{a}$.

Consequently the partial derivatives of the inertia tensor components with respect to the cartesian displacements may easily be deduced. The next result is

$$
J_{\alpha \alpha, S}^{(i)}=\sum_{a}\left(\frac{\partial I_{\alpha \alpha}}{\partial \beta_{a}}\right)_{0} \frac{\partial \beta_{a}}{\partial S_{i}}+\sum_{a}\left(\frac{\partial I_{\alpha \alpha}}{\partial \gamma_{a}}\right)_{0} \frac{\partial \gamma_{a}}{\partial S_{i}}
$$

and

$$
J_{\alpha \beta, S}^{(i)}=\sum_{a}\left(\frac{\partial I_{\alpha \beta}}{\partial \alpha_{a}}\right)_{0} \frac{\partial \alpha_{a}}{\partial S_{i}}+\sum_{a}\left(\frac{\partial I_{\alpha \beta}}{\partial \beta_{a}}\right)_{0} \frac{\partial \beta_{a}}{\partial S_{i}},
$$

where $\alpha, \beta, \gamma$ represents the three cyclic permutations of $x, y, z$; and all the nonvanishing partial derivatives are included. Assume now that the cartesian displacements are linearly dependent on the internal coordinates according to

$x_{a}=\sum_{i} A_{a i}^{x} S_{i}, \quad y_{a}=\sum_{i} A_{a i}^{y} S_{i}, \quad z_{a}=\sum_{i} A_{a i}^{z} S_{i}$.

Then the coefficients $\partial \alpha_{a} / \partial S_{i}$ (for $\alpha=x, y, z$ ) may be identified with $A_{a i}^{\alpha}$, and the final result for the quantities in question reads as given below

$$
\begin{aligned}
& J_{x x, S}^{(i)}=2 \sum_{a} m_{a}\left(Y_{a}^{\mathrm{e}} A_{a i}^{y}+Z_{a}^{\mathrm{e}} A_{a i}^{z}\right), \\
& J_{y y, S}^{(i)}=2 \sum_{a} m_{a}\left(Z_{a}^{\mathrm{e}} A_{a i}^{z}+X_{a}^{\mathrm{e}} A_{a i}^{x}\right), \\
& J_{z z, S}^{(i)}=2 \sum_{a} m_{a}\left(X_{a}^{\mathrm{e}} A_{a i}^{x}+Y_{a}^{\mathrm{e}} A_{a i}^{y}\right), \\
& J_{y z, S}^{(i)}=-\sum_{a} m_{a}\left(Y_{a}^{\mathrm{e}} A_{a i}^{z}+Z_{a}^{\mathrm{e}} A_{a i}^{y}\right),
\end{aligned}
$$

$$
\begin{aligned}
& J_{z x, S}^{(i)}=-\sum_{a} m_{a}\left(Z_{a}^{\mathrm{e}} A_{a i}^{x}+X_{a}^{\mathrm{e}} A_{a i}^{z}\right), \\
& J_{x y, S}^{(i)}=-\sum_{a} m_{a}\left(X_{a}^{\mathrm{e}} A_{a i}^{y}+Y_{a}^{\mathrm{e}} A_{a i}^{x}\right) .
\end{aligned}
$$

\section{Matrix Formulation}

\subsection{Auxiliary matrices}

Define the auxiliary matrices

$\left(\boldsymbol{i}_{m}^{x x}\right)_{a}=\left[\begin{array}{ccc}0 & 0 & 0 \\ 0 & m_{a} & 0 \\ 0 & 0 & m_{a}\end{array}\right],\left(\boldsymbol{i}_{m}^{y y}\right)_{a}=\left[\begin{array}{ccc}m_{a} & 0 & 0 \\ 0 & 0 & 0 \\ 0 & 0 & m_{a}\end{array}\right],\left(\boldsymbol{i}_{m}^{z z}\right)_{a}=\left[\begin{array}{ccc}m_{a} & 0 & 0 \\ 0 & m_{a} & 0 \\ 0 & 0 & 0\end{array}\right]$

and

$\left(\boldsymbol{i}_{m}^{y z}\right)_{a}=\left[\begin{array}{ccc}0 & 0 & 0 \\ 0 & 0 & m_{a} \\ 0 & m_{a} & 0\end{array}\right], \quad\left(\boldsymbol{i}_{m}^{z x}\right)_{a}=\left[\begin{array}{ccc}0 & 0 & m_{a} \\ 0 & 0 & 0 \\ m_{a} & 0 & 0\end{array}\right],\left(\boldsymbol{i}_{m}^{x y}\right)_{a}=\left[\begin{array}{ccc}0 & m_{a} & 0 \\ m_{a} & 0 & 0 \\ 0 & 0 & 0\end{array}\right]$.

The $3 N \times 3 N$ matrices for $a=1,2,3, \ldots, N$, say $\boldsymbol{i}_{m}^{x x}, \ldots, \boldsymbol{i}_{m}^{y z}, \ldots$; are each formed by means of $N$ blocks of one of the above given types. Thus

$$
\boldsymbol{i}_{m}^{\alpha \alpha}=\operatorname{diag}\left[\left(\boldsymbol{i}_{m}^{\alpha \alpha}\right)_{1},\left(\boldsymbol{i}_{m}^{\alpha \alpha}\right)_{2}, \ldots,\left(\boldsymbol{i}_{m}^{\alpha \alpha}\right)_{N}\right]
$$

for $\alpha=x, y$ and $z$. These matrices are truly diagonal. Furtheron we have the three matrices with $3 \times 3$ diagonal blocks, viz.

$$
\boldsymbol{i}_{m}^{\alpha \beta}=\operatorname{diag}\left[\left(\boldsymbol{i}_{m}^{\alpha \beta}\right)_{1},\left(\boldsymbol{i}_{m}^{\alpha \beta}\right)_{2}, \ldots,\left(\boldsymbol{i}_{m}^{\alpha \beta}\right)_{N}\right],
$$

where $\alpha, \beta=x, y$ or $z$. The here defined matrices are connected through the relations

$$
\boldsymbol{i}_{m}^{\alpha \alpha}=\boldsymbol{i}_{m}^{\beta \gamma}\left(\boldsymbol{i}_{m}^{\beta \gamma}\right)^{\prime},
$$

where $\alpha, \beta, \gamma$ represent the three cyclic permutations of $x, y, z$. All the here defined matrices are symmetric. Similar, but skew-symmetric auxiliary matrices have been defined by Meal and $\mathrm{PoLO}^{2}$ in their treatment of Coriolis interaction of vibrationrotation.

We will also need another set of symmetric auxiliary matrices obtained from the above defined set by replacing the atomic masses with unity. We define specifically

$$
\begin{aligned}
& \left(\boldsymbol{i}^{x x}\right)_{a}=\left[\begin{array}{lll}
0 & 0 & 0 \\
0 & 1 & 0 \\
0 & 0 & 1
\end{array}\right], \quad\left(\boldsymbol{i}^{y y}\right)_{a}=\left[\begin{array}{lll}
1 & 0 & 0 \\
0 & 0 & 0 \\
0 & 0 & 1
\end{array}\right],\left(\boldsymbol{i}^{z z}\right)_{a}=\left[\begin{array}{lll}
1 & 0 & 0 \\
0 & 1 & 0 \\
0 & 0 & 0
\end{array}\right] ;
\end{aligned}
$$

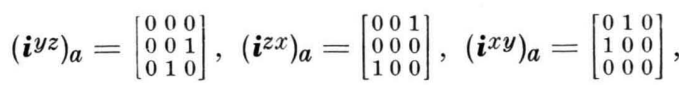

and the $3 N \times 3 N$ matrices formed from these submatrices we shall simply denote $\boldsymbol{i}^{x x}, \ldots, \boldsymbol{i}^{y z}, \ldots$. 


\subsection{Partial derivatives of inertia tensor components: $J$}

Let $\boldsymbol{A}$ be the transformation matrix defined in the usual way by ${ }^{4-6}$

$$
\boldsymbol{X}=\boldsymbol{A} \boldsymbol{S} .
$$

It contains as elements the $A_{a i}^{\alpha}$ coefficients of Eqs. (10) and (11). Now we introduce the column matrix of $3 N$ elements composed of the equilibrium position vector components for all atoms, viz.

$$
\boldsymbol{R}^{\mathrm{e}}=\left\{X_{1}^{\mathrm{e}}, Y_{1}^{\mathrm{e}}, Z_{1}^{\mathrm{e}}, \ldots, X_{N}^{\mathrm{e}}, Y_{N}^{\mathrm{e}}, Z_{N}^{\mathrm{e}}\right\} .
$$

In addition let $\boldsymbol{J}_{\alpha \alpha, S}$ and $\boldsymbol{J}_{\alpha \beta, S}$ denote the column matrices containing as elements $J_{\alpha \alpha, S}^{(i)}$ and $J_{\alpha \beta, S}^{(i)}$ respectively. Hence in terms of the here defined matrices we have

$$
\boldsymbol{J}_{\alpha \alpha, S}=2 \boldsymbol{A}^{\prime} \boldsymbol{i}_{m}^{\alpha \alpha} \boldsymbol{R}^{\mathbf{e}}, \quad \boldsymbol{J}_{\alpha \beta, S}=-\boldsymbol{A}^{\prime} \boldsymbol{i}_{m}^{\alpha \beta} \boldsymbol{R}^{\mathrm{e}} .
$$

These relations (21) are equivalent to the set of Eqs. (11).

We may also express the derivatives with respect to a normal coordinate $\left(Q_{k}\right)$ in matrix notation. In analogy with Eqs. (21) we write

$$
\boldsymbol{J}_{\alpha \alpha, Q}=2 \boldsymbol{a}^{\prime} \boldsymbol{i}_{m}^{\alpha \alpha} \boldsymbol{R}^{\mathrm{e}}, \quad \boldsymbol{J}_{\alpha \beta, Q}=-\boldsymbol{a}^{\prime} \boldsymbol{i}_{m}^{\alpha \beta} \boldsymbol{R}^{\mathrm{e}} .
$$

Here $\boldsymbol{a}$ is defined by $\boldsymbol{X}=\boldsymbol{a} \boldsymbol{Q}$. When $\boldsymbol{L}$ is the familiar normal -coordinate transformation matrix of ${ }^{4}$

one obtains

$$
\boldsymbol{S}=\boldsymbol{L} \boldsymbol{Q}
$$

$$
\boldsymbol{a}=\boldsymbol{A L} .
$$

On combining the relations (21), (22) and (24) one finally obtains the connections

$$
\boldsymbol{J}_{\alpha \alpha, Q}=\boldsymbol{L}^{\prime} \boldsymbol{J}_{\alpha \alpha, S}, \quad \boldsymbol{J}_{\alpha \beta, Q}=\boldsymbol{L}^{\prime} \boldsymbol{J}_{\alpha \beta, S} .
$$

\subsection{Centrifugal distortion constants: $t$}

When employing the matrices defined in the preceding paragraph Eqs. (2) and (3) may be written in matrix notation

$$
t_{\alpha \beta \gamma \delta}=\boldsymbol{J}_{\alpha \beta, Q}^{\prime} \boldsymbol{\sigma} \boldsymbol{J}_{\gamma \delta, Q}=\boldsymbol{J}_{\alpha \beta, S}^{\prime} \boldsymbol{N} \boldsymbol{J}_{\gamma \delta, S} .
$$

Here $\sigma$ is the diagonal matrix of the frequency parameters $\sigma_{k}$; cf. Eq. (4) and ${ }^{6} . \mathbf{N}$ is the compliance matrix ${ }^{3,6}$. The equality of the two expressions in (26) is verified by means of Eqs. (25) and 6

$$
\boldsymbol{N}=\boldsymbol{L} \boldsymbol{\sigma} \boldsymbol{L}^{\prime} .
$$

4 E. B. Wilson, Jr., J. C. Decius and P. C. Cross, Molecular Vibrations, McGraw-Hill, New York 1955.

5 B. N. Cyvin, S. J. Cyvin and L. A. Kristiansen, J. Chem. Phys. 39, 1967 [1963].
The matrix notation of this paragraph may be pursued further. Let $t$ be the complete symmetric tensor (or matrix) defined by the twenty-one distinct quantities of $t_{\alpha \beta \gamma \delta}$ arranged in the following way.

$t=\left[\begin{array}{llllll}t_{x x x x} & t_{x x y y} & t_{x x z z} & t_{x x y z} & t_{x x z x} & t_{x x x y} \\ & t_{y y y y} & t_{y y z z} & t_{y y y z} & t_{y y z x} & t_{y y x y} \\ & & t_{z z z z} & t_{z z y z} & t_{z z z x} & t_{z z x y} \\ & & & t_{y z y z} & t_{y z z} & t_{y z x y} \\ & & & & t_{z x z x} & t_{z x x y} \\ \text { (symmetric) } & & & & t_{x y x y}\end{array}\right]$.

Now let $\boldsymbol{J}_{Q}$ be the matrix with six columns given by $\boldsymbol{J}_{x x, Q}, \boldsymbol{J}_{y y, Q}, \boldsymbol{J}_{z z, Q}, \boldsymbol{J}_{y z, Q}, \boldsymbol{J}_{z x, Q}$ and $\boldsymbol{J}_{x y, Q}$; and similarly for a matrix $\boldsymbol{J}_{S}$. Then

$$
\boldsymbol{t}=\boldsymbol{J}_{Q}^{\prime} \boldsymbol{\sigma} \boldsymbol{J}_{Q}=\boldsymbol{J}_{S}^{\prime} \boldsymbol{N} \boldsymbol{J}_{S}
$$

gives the relations equivalent to (26).

\section{New Relations for Centrifugal Distortion Constants}

\subsection{Preliminary relation}

On combining Eqs. (21) and (26) one obtains

$$
t_{\alpha \beta \gamma \delta}=\left(\boldsymbol{R}^{\mathrm{e}}\right)^{\prime} \boldsymbol{i}_{m}^{\alpha \beta} \boldsymbol{A} \boldsymbol{N} \boldsymbol{A}^{\prime} \boldsymbol{i}_{m}^{\gamma \delta} \boldsymbol{R}^{\mathrm{e}},
$$

where the quantities of $\bar{t}_{\alpha \beta \gamma \delta}$ are closely related to those of $t_{\alpha \beta \gamma \delta}$. As a matter of fact

$$
\begin{array}{ll}
t_{\alpha \alpha \alpha \alpha}=\frac{1}{4} t_{\alpha \alpha \alpha \alpha}, & \bar{t}_{\alpha \alpha \beta \beta}=\frac{1}{4} t_{\alpha \alpha \beta \beta}, \\
t_{\alpha \alpha \alpha \beta}=-\frac{1}{2} t_{\alpha \alpha \alpha \beta}, & t_{\alpha \alpha \beta \gamma}=-\frac{1}{2} t_{\alpha \alpha \beta \gamma}, \\
t_{\alpha \beta \alpha \beta}=t_{\alpha \beta \alpha \beta}, & \bar{t}_{\alpha \beta \beta \gamma}=t_{\alpha \beta \beta \gamma} .
\end{array}
$$

Here $\alpha, \beta$ and $\gamma$ are all different and equal to $x, y$ or $z$. The application of Eq. (30) in practical calculations of centrifugal distortion constants would be to follow closely the method outlined by KIVELson and WILSON ${ }^{1}$; at least in both cases the quantities of $J_{\alpha \beta, S}^{(i)}$ are to be evaluated explicitly. We shall modify the relation (30) in order to develop an easier method for the calculation of the centrifugal distortion constants. Instead of the $J_{\alpha \beta, S}^{(i)}$ quantities there will be others which need to be evaluated, and shall be referred to by the symbol $T_{\alpha \beta, S}^{(i)}$. These new quantities have proved to be constantly simpler than the corresponding $J_{\alpha \beta, S}^{(i)}$, and they are easily developed without the need of an explicit evaluation of the moments of inertia, and neither the $A_{a i}^{\alpha}$ coefficients.

\footnotetext{
6 S. J. Cyvin, Molecular Vibrations and Mean Square
} Amplitudes, Universitetsforlaget, Oslo 1968. 


\subsection{Modified relations}

We employ the well-known relation due to Crawford and Fletcher ${ }^{7}$ :

$$
\boldsymbol{A}=\boldsymbol{m}^{-1} \boldsymbol{B}^{\prime} \boldsymbol{G}^{-1},
$$

where $\boldsymbol{m}^{-1}$ is a diagonal $3 N \times 3 N$ matrix composed of the inverse atomic masses, $\boldsymbol{B}$ is the familiar transformation matrix of

$$
\boldsymbol{S}=\boldsymbol{B} \boldsymbol{X},
$$

and $\boldsymbol{G}$ is still more familiar ${ }^{4}$. After inserting (32) into (30) one obtains the modified relation

$$
\bar{t}_{\alpha \beta \gamma \delta}=\left(\boldsymbol{R}^{\mathrm{e}}\right)^{\prime} \boldsymbol{i}^{\alpha \beta} \boldsymbol{B}^{\prime} \boldsymbol{\Theta} \boldsymbol{B} \boldsymbol{i}^{\gamma \delta} \boldsymbol{R}^{\mathbf{e}},
$$

where $\Theta$ is given by

$$
\boldsymbol{\Theta}=\boldsymbol{G}^{-1} \boldsymbol{N} \boldsymbol{G}^{-1},
$$

and the form of $i^{\alpha \beta}$ is explained at the end of section 2.1.

We define the column matrices $\boldsymbol{T}_{\alpha \beta, S}$ by relations similar to (21), viz.

$$
\boldsymbol{T}_{\alpha \alpha, s}=2 \boldsymbol{B} \boldsymbol{i}^{\alpha \alpha} \boldsymbol{R}^{\mathbf{e}}, \quad \boldsymbol{T}_{\alpha \beta, S}=-\boldsymbol{B} \boldsymbol{i}^{\alpha \beta} \boldsymbol{R}^{\mathrm{e}} .
$$

Hence

$$
t_{\alpha \beta \gamma \delta}=\boldsymbol{T}_{\alpha \beta, S}^{\prime} \boldsymbol{\Theta} \boldsymbol{T}_{\gamma \delta, S},
$$

which displays its analogy with Eq. (26). We may also define the $\boldsymbol{T}_{S}$ matrix (in analogy with $\boldsymbol{J}_{S}$ ) as composed of the six columns $\boldsymbol{T}_{x x, s}, \ldots, \boldsymbol{T}_{x y, S}$. Then

$$
t=\boldsymbol{T}_{S}^{\prime} \boldsymbol{\Theta} \boldsymbol{T}_{S}
$$

in analogy with Eq. (29).

\subsubsection{Properties of the matrices $\boldsymbol{\Theta}$ and $\boldsymbol{\Phi}$}

In this section we shall discuss briefly the matrix $\boldsymbol{\Theta}$ of Eq. (35) and its inverse $\boldsymbol{\Phi} ; \boldsymbol{\Theta}=\boldsymbol{\Phi}^{-1}$. These matrices are given by

$$
\boldsymbol{\Phi}=\boldsymbol{G} \boldsymbol{F} \boldsymbol{G}, \quad \boldsymbol{\Theta}=\boldsymbol{M} \boldsymbol{N} \boldsymbol{M},
$$

where $\boldsymbol{M}=\boldsymbol{G}^{-1}$ and $\boldsymbol{N}=\boldsymbol{F}^{-1}$, in consistence with the notation of $\mathrm{Crvin}^{6}$. It is clear that the matrices $\boldsymbol{\Phi}$ and $\boldsymbol{\Theta}$ are obtainable from the usual normalcoordinate analysis of molecular vibrations; their elements could in fact be used as potential constants instead of the force constants $\left(F_{i j}\right)$ or compliants $\left(N_{i j}\right)$. In terms of the $\boldsymbol{L}$ matrix and its inverse, $\boldsymbol{K}=\boldsymbol{L}^{-1}{ }^{6}$, one has

$$
\boldsymbol{\Phi}=\boldsymbol{L} \boldsymbol{\lambda} \boldsymbol{L}^{\prime}, \quad \boldsymbol{\Theta}=\boldsymbol{K}^{\prime} \boldsymbol{\sigma} \boldsymbol{K} .
$$

7 B. L. Crawford, Jr. and W. H. Flechter, J. Chem. Phys. 19,141 [1951].
The matrix $\sigma$ has already been encountered; cf. Eq. (26), and $\sigma=\lambda^{-1}$. Hence $\lambda$ is the well-known diagonal matrix with elements $\lambda_{k}=4 \pi^{2} c^{2} \omega_{k}{ }^{2}$.

We also give some secular equations for $\boldsymbol{\Phi}$ and $\boldsymbol{\Theta}$ :

$$
\boldsymbol{\Phi} \boldsymbol{M L}=\boldsymbol{L} \boldsymbol{\lambda}, \quad G \boldsymbol{\Theta} \boldsymbol{L}=\boldsymbol{L} \boldsymbol{\sigma} .
$$

Notice that $\boldsymbol{\Phi} \boldsymbol{M}=\boldsymbol{G} \boldsymbol{F}$, and $\boldsymbol{G} \boldsymbol{\Theta}=\boldsymbol{N} \boldsymbol{M}$. The relations (41) yield the following secular-determinant equations.

$$
\left|\boldsymbol{\Phi} \boldsymbol{M}-\lambda_{k} \boldsymbol{E}\right|=0, \quad\left|\boldsymbol{G} \boldsymbol{\Theta}-\sigma_{k} \boldsymbol{E}\right|=0 .
$$

3.2.2. Properties of the $\boldsymbol{T}_{S}$ matrix elements

The column matrices $\boldsymbol{T}_{\alpha \beta, S}$ (of which the $\boldsymbol{T}_{S}$ matrix is composed) constitute an essential part of the relation (37). Hence the properties of their elements are important in our discussion of the calculations of centrifugal distortion constants. In consistence with Eqs. (36) the elements in question are given by equations similar to (11):

$$
\begin{aligned}
T_{x x, S}^{(i)} & =2 \sum_{a}\left(Y_{a}^{\mathrm{e}} B_{i a}^{y}+Z_{a}^{\mathrm{e}} B_{i a}^{z}\right), \\
T_{y y, S}^{(i)} & =2 \sum_{a}\left(Z_{a}^{\mathrm{e}} B_{i a}^{z}+X_{a}^{\mathrm{e}} B_{i a}^{x}\right), \\
T_{z z, S}^{(i)} & =2 \sum_{a}\left(X_{a}^{\mathrm{e}} B_{i a}^{x}+Y_{a}^{\mathrm{e}} B_{i a}^{y}\right), \\
T_{y z, S}^{(i)} & =-\sum_{a}\left(Y_{a}^{\mathrm{e}} B_{i a}^{z}+Z_{a}^{\mathrm{e}} B_{i a}^{y}\right), \\
T_{z x, S}^{(i)} & =-\sum_{a}\left(Z_{a}^{\mathrm{e}} B_{i a}^{x}+X_{a}^{\mathrm{e}} B_{i a}^{z}\right), \\
T_{x y, S}^{(i)} & =-\sum_{a}\left(X_{a}^{\mathrm{e}} B_{i a}^{y}+Y_{a}^{\mathrm{e}} B_{i a}^{x}\right) .
\end{aligned}
$$

The notation used here for $\boldsymbol{B}$ matrix elements is explained by

$$
S_{i}=\sum_{a}\left(B_{i a}^{x} x_{a}+B_{i a}^{y} y_{a}+B_{i a}^{z} z_{a}\right) .
$$

It seems clear that the quantities of Eqs. (43) are easier to evaluate than those of Eqs. (11). The former ones, $T_{\alpha \beta, S}^{(i)}$, require the $\boldsymbol{B}$ matrix elements, which are equivalent to Wilson's $s$ vectors ${ }^{4-6}$, while the latter ones, $J_{\alpha \beta, S}^{(i)}$, require the $\boldsymbol{A}$ matrix elements, which are equivalent to PoLo's $\rho^{\circ}$ vectors ${ }^{8}$. These latter vectors are designated $\boldsymbol{t}^{\circ}$ in Cyvin's book ${ }^{6}$. For a special account on the vector methods, see B. N. Crvin et al. 5 .

There is another property which facilitates the derivation of $T_{\alpha \beta, S}^{(i)}$ elements. In Eqis. (43), namely, the equilibrium position vector components need

8 S. R. Polo, J. Chem. Phys. 24, 1133 [1956]. 
not necessarily refer to the center of gravity of the molecule as their origin, as is required in Eqs. (11). This feature is proved in the following. Let $(\bar{X}, \bar{Y}, Z)$ represent a new coordinate system, in which the molecule's center of gravity has the coordinates $\left(\bar{X}_{0}, \bar{Y}_{0}, Z_{0}\right)$. But the axes of the new system should be parallel to a set of principal axes. Hence one has

$$
\boldsymbol{R}^{\mathbf{e}}=\overline{\boldsymbol{R}}_{\mathbf{0}}+\overline{\boldsymbol{R}}^{\mathbf{e}}
$$

for the transformation of the equilibrium positionvector matrix defined by Eq. (20). In the above equation (45) $\overline{\boldsymbol{R}}_{0}$ is the column matrix with $3 \mathrm{~N}$ elements given by

$$
\overline{\boldsymbol{R}}_{0}=\left\{\bar{X}_{0}, \bar{Y}_{0}, \bar{Z}_{0}, \ldots, \bar{X}_{0}, \bar{Y}_{0}, \bar{Z}_{0}\right\} .
$$

After inserting (45) into (36) it is found

$$
\begin{aligned}
& \overline{\boldsymbol{T}}_{\alpha \alpha, S}=\mathbf{2} \boldsymbol{B} \boldsymbol{i}^{\alpha \alpha} \overline{\boldsymbol{R}} \mathbf{e}=\boldsymbol{T}_{\alpha \alpha, S}-2 \boldsymbol{B} \boldsymbol{i}^{\alpha \alpha} \overline{\boldsymbol{R}}_{\mathbf{0}}, \\
& \overline{\boldsymbol{T}}_{\alpha \beta, S}=-\boldsymbol{B} \boldsymbol{i}^{\alpha \beta} \overline{\boldsymbol{R}} \mathbf{e}=\boldsymbol{T}_{\alpha \beta, S}+\boldsymbol{B} \boldsymbol{i}^{\alpha \beta} \overline{\boldsymbol{R}}_{\mathbf{0}} .
\end{aligned}
$$

But it is found

$$
\boldsymbol{B} \boldsymbol{i}^{\alpha \alpha} \overline{\boldsymbol{R}}_{0}=\boldsymbol{O}, \quad \boldsymbol{B} \boldsymbol{i}^{\alpha \beta} \overline{\boldsymbol{R}}_{0}=\boldsymbol{O}
$$

when these matrix products are evaluated. This fact is due to the property of vanishing sums of $s$ vectors 9,10 . For our purpose the same property may be expressed in terms of $\boldsymbol{B}$-matrix elements as

$$
\sum_{a} B_{i a}^{x}=\sum_{a} B_{i a}^{y}=\sum_{a} B_{i a}^{z}=0 .
$$

This completes the proof since it follows from Eqs. (47) and (48):

$$
\overline{\boldsymbol{T}}_{\alpha \alpha, S}=\boldsymbol{T}_{\alpha \alpha, S}, \quad \overline{\boldsymbol{T}}_{\alpha \beta, S}=\boldsymbol{T}_{\alpha \beta, S} .
$$

It has already been demonstrated how the centrifugal distortion constants can be obtained by means of the $T_{a \beta, S}^{(i)}$ elements [see, e.g. Eq. (37)], without explicit evaluation of the partial derivatives $J_{\alpha \beta, S}^{(i)}$. But these two types of quantities are naturally interconnected, and as a matter of fact the latter can be derived from the former. On combining Eqs. (21), (32) and (36) it has been found

$$
\boldsymbol{J}_{\alpha \alpha, S}=\boldsymbol{G}^{-1} \boldsymbol{T}_{\alpha \alpha, S}, \quad \boldsymbol{J}_{\alpha \beta, S}=\boldsymbol{G}^{-1} \boldsymbol{T}_{\alpha \beta, S} .
$$

\section{Example: Bent Symmetrical $\mathrm{XY}_{2}$ Model}

\subsection{Quantities of $J_{\alpha \beta, S}^{(i)}$ and $T_{\alpha \beta, S}^{(i)}$}

The bent symmetrical $X Y_{2}$ molecule model has been used as example also by KIVELSon and WILsoN ${ }^{1}$. In their discussion of symmetry properties they derived rules for vanishing of $J_{\alpha \beta, S}^{(i)}$ elements.

Exactly the same rules apply to our $T_{\alpha \beta, S}^{(i)}$ elements. We have recalculated the derivatives of inertia tensor components using a different orientation of the molecule model with respect to the principal axes than used by KIVELSon and WILSON ${ }^{1}$. This we have done because we insist on using the recommendations of MULLIKEN 11 and standardized orientation of symmetry coordinates, which are fully discussed previously 12 . Our result reads:

$$
\begin{aligned}
J_{x x, S}^{(1)}\left(A_{1}\right)= & 8^{1 / 2} M^{-1} m_{\mathrm{Y}} R\left(m_{\mathrm{X}}+2 m_{\mathrm{Y}} \sin ^{2} A\right), \\
J_{x x, S}^{(2)}\left(A_{1}\right)= & 2 M^{-1} m_{\mathrm{Y}}^{2} R \sin 2 A \\
J_{y y, S}^{(1)}\left(A_{1}\right)= & 8^{1 / 2} M^{-1} m_{\mathrm{X}} m_{\mathrm{Y}} R \cos ^{2} A \\
J_{y y, S}^{(2)}\left(A_{1}\right)= & -M^{-1} m_{\mathrm{X}} m_{\mathrm{Y}} R \sin 2 A \\
J_{z z, S}^{(1)}\left(A_{1}\right)= & 8^{1 / 2} m_{\mathrm{Y}} R \sin ^{2} A \\
J_{z z, S}^{(2)}\left(A_{1}\right)= & m_{\mathrm{Y}} R \sin 2 A \\
J_{y z, S}\left(B_{2}\right)= & 2^{1 / 2} m_{\mathrm{X}} m_{\mathrm{Y}} \\
& \cdot\left(m_{\mathrm{X}}+2 m_{\mathrm{Y}} \sin ^{2} A\right)^{-1} R \sin 2 \mathrm{~A} .
\end{aligned}
$$

For explanation of the applied symbols, see Fig. 1. The corresponding quantities of $T_{\alpha \beta, S}^{(i)}$ are found to be considerably simpler. They are collected in Table 1.

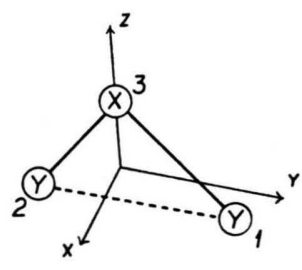

(a)

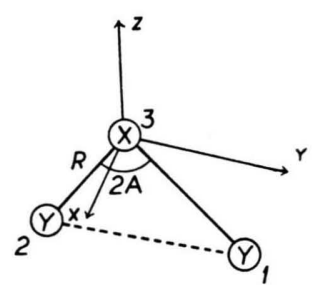

(b)

Fig. 1. Bent symmetrical $\mathrm{XY}_{2}$ molecular model with the origin of cartesian axes in (a) the center of gravity of the molecule, and (b) the atom $\mathrm{X}$. Equilibrium position vectors:

(a)

$$
\left\{\begin{array}{ccc}
0, & R \sin A, & -M^{-1} m_{x} R \cos A \\
\{0, & -R \sin A, & -M^{-1} m_{x} R \cos A \\
0, & 0, & 2 M^{-1} m_{\mathrm{Y}} R \cos A
\end{array}\right\}
$$

The symbols $m_{\mathrm{X}}$ and $m_{\mathrm{Y}}$ are used to denote the atomic masses of $\mathrm{X}$ and $\mathrm{Y}$, respectively, and $M$ is the total mass of the molecule, $\boldsymbol{M}=m_{\mathrm{X}}+2 m_{\mathrm{Y}}$. For the principal moments of inertia at equilibrium:

$$
I_{x x}^{\mathrm{e}}=I_{y y}^{\mathrm{e}}+I_{z z}^{\mathrm{e}}=2 M^{-1} R^{2} m_{\mathrm{Y}}\left(m_{\mathrm{X}}+2 m_{\mathrm{Y}} \sin ^{2} A\right) .
$$

A standard set of symmetry coordinates is specified elsewhere ${ }^{12}$.

9 R. J. Malhiot and S. M. Ferigle, J. Chem. Phys. 22, 717 [1954].

10 R. J. Malhiot and S. M. Ferigle, J. Chem. Phys. 23, 30 [1955].

11 R. S. Mulliken, J. Chem. Phys. 23, 1997 [1955].

12 S. J. Cyvin, J. Brunvoll, B. N. Cyvin, I. Elvebredd and G. Hagen, Mol. Phys. 14, 43 (1968). 


\begin{tabular}{cccc}
\hline $\boldsymbol{T}_{\alpha \alpha, S}$ & $x x$ & $y y$ & $z z$ \\
\hline$S_{1}\left(A_{1}\right)$ & $8^{1 / 2} R$ & $8^{1 / 2} R \cos ^{2} A$ & $8^{1 / 2} R \sin ^{2} A$ \\
$S_{2}\left(A_{1}\right)$ & 0 & $-2 R \sin 2 A$ & $2 R \sin 2 A$ \\
\hline$T_{\alpha \beta, S}$ & \multicolumn{3}{|}{$y z$} \\
\hline$S\left(B_{2}\right)$ & \multicolumn{3}{c}{$2^{1 / 2} R \sin 2 A$} \\
\hline
\end{tabular}

Table 1. Tabulation of the quantities $T_{\alpha \beta, S}^{(i)}$ for the bent symmetrical $\mathrm{XY}_{2}$ model.

The connection between the $\boldsymbol{J}_{S}$ and $\boldsymbol{T}_{S}$ elements given by Eq. (51) may easily be verified in the present case, using the $\boldsymbol{G}^{-1}$ matrix elements tabulated elsewhere ${ }^{6}$; here the species designation $B_{1}$ should be changed to $B_{2}$ in order to conform our new orientation.

\subsection{The applied vector components}

The above results of Eqs. (52) and Table 1 were derived by means of the following vector components. Firstly the position vector components given in the legend of Fig. 1 (a) and (b). In the case of the quantities in Table 1 we could use the simple position vectors of Fig. 1 (b), where the origin is placed in the position of atom $X$ rather than the center of gravity of the molecule. Exactly the same choice of the cartesian axes has been made previously ${ }^{12}$. Secondly, the $\boldsymbol{J}_{S}$ and $\boldsymbol{T}_{S}$ elements were derived by means of the $\boldsymbol{A}$ and $\boldsymbol{B}$ matrix elements, respectively. These elements are equivalent to the components of the $\boldsymbol{t}^{\circ}$ (in PoLo's ${ }^{8}$ notation $\rho^{\circ}$ ) and $\boldsymbol{s}$ vectors, respectively. All these vectors for the molecular model in question are given in the book of Cyvin ${ }^{6}$, but they should be changed according to the new standard orientation of the cartesian $\operatorname{axes}^{12}$. Therefore we give in Table 2 the $\boldsymbol{s}$ and $\boldsymbol{t}^{\circ}$ vectors which are consistent with the new orientation also shown in Fig. 1.

\subsection{Centrifugal distortion constants}

With the aid of the elements from Table 1 the centrifugal distortion constants may now be evaluated in terms of $\boldsymbol{\Theta}$ matrix elements according to the relation (37) or (38). The results are simple enough to be given here.

$$
\begin{gathered}
t_{x x x x}=8 R^{2} \Theta_{11}, \\
t_{y y y y}=8 R^{2} \Theta_{11} \cos ^{4} A-8 \sqrt{2} R^{2} \Theta_{12} \sin 2 A \cos ^{2} A \\
\quad+4 R^{2} \Theta_{22} \sin ^{2} 2 A, \\
t_{z z z z}=8 R^{2} \Theta_{11} \sin ^{4} A+8 \sqrt{2} R^{2} \Theta_{12} \sin 2 A \sin ^{2} A \\
\quad+4 R^{2} \Theta_{22} \sin ^{2} 2 A, \\
t_{x x y y}=8 R^{2} \Theta_{11} \cos ^{2} A-4 \sqrt{2} R^{2} \Theta_{12} \sin 2 A, \quad(53) \\
t_{x x z z}=8 R^{2} \Theta_{11} \sin ^{2} A+4 \sqrt{2} R^{2} \Theta_{12} \sin 2 A, \\
t_{y y z z}=2 R^{2} \Theta_{11} \sin ^{2} 2 A+4 \sqrt{2} R^{2} \Theta_{12} \sin 2 A \cos 2 A \\
\quad-4 R_{22} \sin ^{2} 2 A, \\
t_{y z y z}=2 R^{2} \Theta_{33} \sin ^{2} 2 A .
\end{gathered}
$$

KIVELSON and WILSON ${ }^{1}$ have pointed out the dependences between six of the constants in Eqs. (53). They arise from the relations

$$
T_{x x, S}^{(i)}=T_{y y, S}^{(i)}+T_{z z, S}^{(i)},
$$

and likewise for the $J_{\alpha \alpha, S}^{(i)}$ quantities. These relations may be traced back to $I_{x x}^{\mathrm{e}}=I_{y y}^{\mathrm{e}}+I_{z z}^{\mathrm{e}}$, which holds on account of the planarity of the molecule. In our notation the dependences between appropriate centrifugal distortion constants read:

$$
\begin{aligned}
& t_{x x x x}=t_{y y y y}+t_{z z z z}+2 t_{y y z z}, \\
& t_{x x y y}=t_{y y y y}+t_{y y z z}, \quad t_{x x z z}=t_{z z z z}+t_{y y z z} .
\end{aligned}
$$

On combining these relations one also obtains: $t_{x x x x}=t_{x x y y}+t_{x x z z}$.

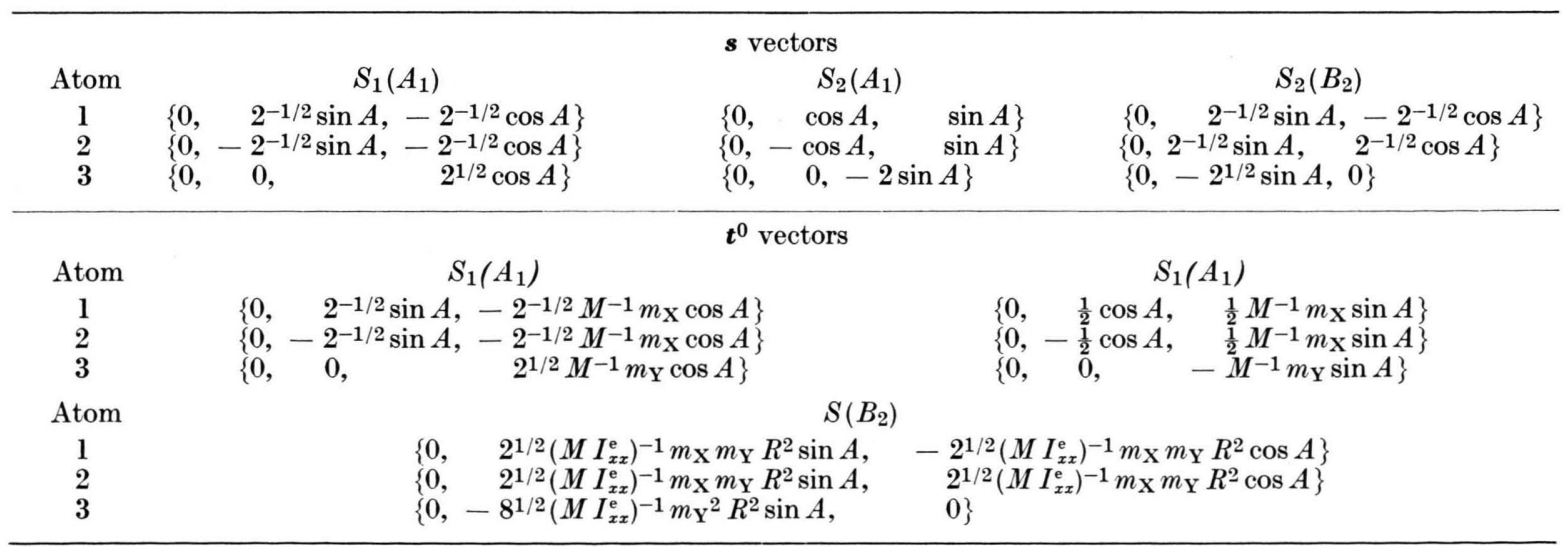

Table 2. $\boldsymbol{s}$ and $\boldsymbol{t}^{0}$ vectors for the bent symmetrical $X Y_{2}$ model. 


\section{Numerical Computations}

In order to provide some numerical examples we have calculated the here considered centrifugal distortion constants for a number of bent symmetrical $X Y_{2}$ molecules. The structural parameters and force constants (with minor adjustments) are taken from OKa and MoRino ${ }^{13}$. Other computations for the same molecules (with the same force fields) are reported elsewhere ${ }^{6}$. For the sake of brevity we have not included here all the isotopic varieties of the compounds, which are found in the cited works ${ }^{13,6}$.

\begin{tabular}{lcccrrc}
\hline Molecule & \multicolumn{1}{c}{ Species } & $\boldsymbol{F}$ & \multicolumn{1}{c}{$\boldsymbol{N}$} & \multicolumn{1}{c}{$\boldsymbol{\Phi}$} & $\boldsymbol{\Theta}$ \\
\hline $\mathrm{H}_{2}{ }^{16} \mathrm{O}$ & $A_{1}$ & 11 & 8.354 & 0.122 & 8.960 & 0.112 \\
& & 12 & 0.331 & -0.053 & -0.144 & 0.005 \\
& & 22 & 0.761 & 1.337 & 3.425 & 0.292 \\
$\mathrm{D}_{2}{ }^{16} \mathrm{O}$ & $B_{2}$ & & 8.353 & 0.120 & 9.565 & 0.105 \\
& & 11 & 8.354 & 0.122 & 2.439 & 0.421 \\
& & 12 & 0.331 & -0.053 & -0.254 & 0.107 \\
& $B_{2}$ & & 0.761 & 1.337 & 1.001 & 1.026 \\
$\mathrm{H}_{2}{ }^{32 \mathrm{~S}}$ & $A_{1}$ & 11 & 4.273 & 0.235 & 4.456 & 0.224 \\
& & 12 & 0.093 & -0.051 & -0.037 & 0.005 \\
& & 22 & 0.426 & 2.358 & 1.780 & 0.562 \\
$\mathrm{D}_{2}{ }^{32} \mathrm{~S}$ & $B_{2}$ & & 4.273 & 0.234 & 4.484 & 0.223 \\
& $A_{1}$ & 11 & 4.273 & 0.235 & 1.181 & 0.854 \\
& & 12 & 0.093 & -0.051 & -0.068 & 0.121 \\
& $B_{2}$ & & 4.273 & 0.234 & 1.195 & 0.837 \\
\hline
\end{tabular}

Table 3. Vibrational constants for $\mathrm{H}_{2} \mathrm{O}$ and $\mathrm{H}_{2} \mathrm{~S}$ molecules. Units: $F$ [mdyne $\left.\AA^{-1}\right], N\left[\AA\right.$ mdyne $\left.^{-1}\right], \Phi$ [mdyne $\AA^{-1}$ $\left.\mathrm{Amu}^{-2}\right], \quad \Theta\left[\mathrm{Amu}^{2} \AA\right.$ mdyne $\left.{ }^{-1}\right]$.

Table 3 shows the force constants $(F)$ and compliants $(N)$, along with the additional vibrational constants $\Phi$ and $\Theta$ for two pairs of isotopic molecules $\left(\mathrm{H}_{2} \mathrm{O}\right.$ and $\left.\mathrm{H}_{2} \mathrm{~S}\right)$. The force constants are as usual assumed to be the same for isotopic molecules. As a consequence the compliants are the same too. But this is of course not true for the $\Phi$ and $\Theta$ constants. As to the interaction constants $\Phi_{i j}$ and $\Theta_{i j}(i \neq j)$ it may be concluded from the present work that they cannot be neglected in general.

Table 4 gives the four independent $t_{\alpha \beta \gamma \delta}$ constants for the thirteen molecules treated in the present work. The remaining nonvanishing $t_{\alpha \beta \gamma \delta}$ constants are obtainable from Eqs. (54). For the sake of clarity we also give here the nonvanishing $\boldsymbol{T}_{S}$ elements (cf. Table 1) for the first molecules listed (viz. $\mathrm{H}_{2} \mathrm{O} ; R=0.9572 \AA, 2 A=104^{\circ} 32^{\prime}$ ). They are (in $\AA$ ): $T_{x x, S}^{(1)}\left(A_{1}\right)=2.707, T_{y y, S}^{(1)}\left(A_{1}\right)=1.014$,

$$
\begin{aligned}
T_{z z, S}^{(1)}\left(A_{1}\right) & =1.693, T_{y y, S}^{(2)}=-T_{z z, S}^{(2)}\left(A_{1}\right) \\
& =-1.853, T_{y z, S}\left(B_{2}\right)=1.310 .
\end{aligned}
$$

\begin{tabular}{lcccc}
\hline Molecule & \multicolumn{1}{c}{$t_{\text {yyyy }}$} & \multicolumn{1}{c}{$t_{z z z z}$} & \multicolumn{1}{l}{$t_{y y z z}$} & $t_{y z y z}$ \\
\hline $\mathrm{H}_{2}{ }^{16} \mathrm{O}$ & 1.101 & 1.353 & -0.817 & 0.180 \\
$\mathrm{D}_{2}{ }^{16} \mathrm{O}$ & 3.556 & 5.403 & -2.936 & 0.623 \\
$\mathrm{H}_{2}{ }^{32 \mathrm{~S}}$ & 4.645 & 4.861 & -3.170 & 0.785 \\
$\mathrm{D}_{2}{ }^{32 \mathrm{~S}}$ & 16.536 & 19.414 & -11.952 & 2.947 \\
$\mathrm{H}_{2}{ }^{80 \mathrm{Se}}$ & 7.746 & 8.235 & -5.392 & 1.296 \\
$\mathrm{D}_{2}{ }^{80 \mathrm{Se}}$ & 29.47 & 32.89 & -21.02 & 5.048 \\
${ }^{14} \mathrm{~N}^{16} \mathrm{O}_{2}$ & 17.14 & 288.76 & -36.90 & 3.359 \\
${ }^{16} \mathrm{O}_{3}$ & 36.78 & 435.81 & -48.51 & 15.377 \\
${ }^{32} \mathrm{~S}^{16} \mathrm{O}_{2}$ & 138.6 & 675.4 & -201.8 & 25.97 \\
${ }^{35} \mathrm{Cl}^{16} \mathrm{O}_{2}$ & 215.7 & 936.2 & -282.4 & 43.67 \\
${ }^{19} \mathrm{~F}_{2}{ }^{16} \mathrm{O}$ & 67.43 & 1445.8 & -56.39 & 69.31 \\
${ }^{35} \mathrm{Cl}_{2}{ }^{16} \mathrm{O}$ & 367.1 & 6908 & -976.5 & 80.66 \\
${ }^{35} \mathrm{Cl}_{2}{ }^{32} \mathrm{~S}$ & 1648.2 & 22994 & -3796 & 673.6
\end{tabular}

Table 4. $t_{\alpha \beta \gamma \delta}$ constants for bent symmetrical $\mathrm{XY}_{2}$ molecules. Units: $\mathrm{Amu}^{2} \AA^{3}$ mdyne ${ }^{-1}$.

\begin{tabular}{crrrr}
\hline$\left[10^{-4} \mathrm{~cm}^{-1}\right]$ & \multicolumn{1}{c}{$\mathrm{H}_{2}{ }^{16} \mathrm{O}$} & \multicolumn{1}{c}{$\mathrm{D}_{2}{ }^{16} \mathrm{O}$} & \multicolumn{1}{c}{$\mathrm{H}_{2}{ }^{32} \mathrm{~S}$} & $\mathrm{D}_{2}{ }^{32} \mathrm{~S}$ \\
\hline$\tilde{\boldsymbol{\tau}}_{x x x x}$ & 0.589 & 0.160 & 0.157 & 0.042 \\
$\tilde{\boldsymbol{\tau}}_{y y y y}$ & 54.495 & 16.866 & 4.902 & 1.377 \\
$\tilde{\boldsymbol{\tau}}_{z z z z z}$ & 5.353 & 1.342 & 2.954 & 0.740 \\
$\tilde{\boldsymbol{\tau}}_{x x y y}$ & 1.689 & 0.308 & 0.338 & 0.078 \\
$\tilde{\boldsymbol{\tau}}_{x x z z}$ & 0.903 & 0.280 & 0.294 & 0.086 \\
$\tilde{\boldsymbol{\tau}}_{y y z z}$ & -11.442 & -3.187 & -2.538 & -0.673 \\
$\tilde{\boldsymbol{\tau}}_{y z y z}$ & 2.513 & 0.676 & 0.629 & 0.166 \\
\hline
\end{tabular}

Table 5. $\tilde{\tau}_{\alpha \beta \gamma \delta}$ constants in $10^{-4} \mathrm{~cm}^{-1}$ units for $\mathrm{H}_{2} \mathrm{O}$ and $\mathrm{H}_{2} \mathrm{~S}$ molecules.

For two pairs of isotopic molecules (cf. also Table 3) we give in Table 5 the results for $\tilde{\tau}_{\alpha \beta \gamma \delta}$ in $10^{-4} \mathrm{~cm}^{-1}$ units. These quantities we define as proportional to the $\tau_{\alpha \beta \lambda \delta}$ 's of Eq. (1); specifically

$$
\tilde{\tau}_{\alpha \beta \gamma \delta}=-\frac{h^{3}}{256 \pi^{4} c} \tau_{\alpha \beta \gamma \delta} .
$$

The pure rotational spectrum of water vapour has recently been reinvestigated by $\mathrm{HALL}_{\mathrm{AL}}$ and DowLING ${ }^{14}$. Their quantities of $T_{\alpha \beta \gamma \delta}{ }^{14,15}$ correspond to $-16 \tilde{\tau}_{\alpha \beta \gamma \delta}$ in our notation. In Table 6 we finally give the observed 14 and calculated $T_{\alpha \beta \gamma \delta}$ constants for $\mathrm{H}_{2}{ }^{16} \mathrm{O}$. Both our calculated values and those of the cited work ${ }^{14}$ show quite good agreement with the observed ${ }^{14}$ values.

\begin{tabular}{lcccr}
\hline [cm $\left.{ }^{-1}\right]$ & \multicolumn{2}{c}{ Observed } & \multicolumn{2}{c}{ Calculated } \\
& \multicolumn{2}{c}{$\left[{ }^{14}\right]$} & {$[14]$} & \multicolumn{1}{c}{ Present } \\
\hline$T_{x x x x}$ & -0.00107 & \pm 0.00027 & -0.00093 & -0.00094 \\
$T_{y y y y}$ & -0.1084 & \pm 0.0012 & -0.1088 & -0.08719 \\
$T_{z z z z z}$ & -0.0083 & \pm 0.0012 & -0.00672 & -0.00856 \\
$T_{x x y y}$ & -0.00491 & & -0.00515 & -0.00270 \\
$T_{x x z z}$ & -0.00108 & & -0.00077 & -0.00144 \\
$T_{y y z z}$ & 0.01985 & & 0.01846 & 0.01831 \\
$T_{y z y z}$ & & & & -0.00402 \\
\hline
\end{tabular}

Table 6. $T_{\alpha \beta \gamma \delta}$ constants in $\mathrm{cm}^{-1}$ units for $\mathrm{H}_{2}{ }^{16} \mathrm{O}$.

13 T. Oka and Y. Morino, J. Mol. Spectry. 8, 9 [1962].

14 R. T. Hall and J. M. Dowling, J. Chem. Phys. 47, 2454 (1967).

15 P. E. Fraley and K. N. RaO, J. Mol. Spectry. 19, 131 [1966]. 